Research Paper

\title{
RNFI 14 Silencing Inhibits the Proliferation and Metastasis of Gastric Cancer
}

\author{
Zongfeng Feng ${ }^{1,2^{*}}$, Leyan $\mathrm{Li}^{2,3^{*}}$, Qingwen Zeng ${ }^{1,2^{*}}$, Yang Zhang ${ }^{1,2^{*}}$, Yi Tu${ }^{1,4}$, Wenzheng Chen ${ }^{1,2}$, Xufeng Shu ${ }^{1,2}$, \\ Ahao $\mathrm{Wu}^{1,2}$, Jianbo Xiong ${ }^{1,2 \bowtie}$, Yi Cao ${ }^{1,2}{ }^{\circledR}$, Zhengrong $\mathrm{Li}^{1,2 \llbracket}$
}

1. Department of General Surgery, the First Affiliated Hospital of Nanchang University, Nanchang, China.

2. Laboratory of Digestive Surgery, Nanchang University, Nanchang, China.

3. Queen Mary School, Medical Department of Nanchang University, Nanchang, China.

4. Department of Pathology, the First Affiliated Hospital of Nanchang University, Nanchang, China.

*These authors contributed equally to this work.

$\triangle$ Corresponding authors: Zhengrong Li, Department of General Surgery, the First Affiliated Hospital of Nanchang University. No. 17, Yongwai Zheng Road, Nanchang 330006, Jiangxi Province, People's Republic of China. E-mail: lzr13@foxmail.com; Yi Cao, Department of General Surgery, the First Affiliated Hospital of Nanchang University, No.17 Yongwai Zheng Road, Nanchang 330006, Jiangxi Province, People's Republic of China. E-mail: doctorcaoyi@126.com; Jianbo Xiong, Department of General Surgery, the First Affiliated Hospital of Nanchang University, No.17 Yongwai Zheng Road, Nanchang 330006, Jiangxi Province, People's Republic of China. E-mail: Xiongjianbo2017@foxmail.com.

() The author(s). This is an open access article distributed under the terms of the Creative Commons Attribution License (https://creativecommons.org/licenses/by/4.0/). See http://ivyspring.com/terms for full terms and conditions.

Received: 2021.04.25; Accepted: 2021.09.12; Published: 2022.01.01

\begin{abstract}
RNF114 (E3 ubiquitin ligase RING finger protein 114) was first identified as a zinc-binding protein that promotes psoriasis development; however, its role in gastric cancer is still unclear. We explored the relationship between RNFI 44 and gastric cancer using bioinformatics and molecular biology techniques. The results showed that RNF114 was highly expressed in gastric cancer and negatively correlated with the patient's prognosis. Functional assays suggested that RNFI 14 silencing suppressed the proliferation and metastasis of gastric cancer cells to a certain extent. Further studies showed that RNFI I 4 expression was potentially targeted by miR-218-5p and methylation modification, and mediated downstream EGR 1 (early growth response 1) degradation by the ubiquitylation approach. Together, the present results highlight the detrimental effects of RNFI l 4 overexpression in gastric cancer and contribute to a better understanding of the mechanisms underlying RNFI 14 functionality.
\end{abstract}

Key words: RNF114, EGR1, miR-218-5p, methylation, ubiquitylation, gastric cancer

\section{Introduction}

In 2020, gastric cancer (GC) was the fifth most commonly diagnosed malignant tumor and the third leading cause of death in numerous countries, especially the coastal countries of Southeast Asia, with more than 1 million newly diagnosed cases and an estimated 783,000 deaths [1]. Although surgical and chemotherapy treatments have constantly improved, the survival rate of patients with GC remains relatively low $[2,3]$. Therefore, it is necessary to uncover the epigenetic mechanism of GC occurrence and development.

RNF114, also known as ZNF228 or ZNF313, is a member of the zinc-binding protein family $[4,5]$. The RNF114 gene is located on chromosome 20q13.13, which is recognized as a common gene amplification region [6]. RNF114 encodes a protein containing both $\mathrm{C} 2 \mathrm{H} 2$ and RING finger zinc-binding domains, which binds to ubiquitin via the C-terminal ubiquitin interaction motif (UIM) and exerts E3 ligase activity through the N-terminal RING domain [7]. The RNF114 protein is expressed widely in many normal tissues, such as the heart, liver, and kidney; however, it is decreased in the muscle, lung, and placenta [8]. The RNF114 protein is a novel determinant of psoriasis susceptibility, and its overexpression is a crucial indicator of epithelial inflammation [9-11]. Boren et al. [12] reported that RNF114 mediates osteoclastogenesis via the RANKL/RANK/TRAF6 signaling pathway. Huang et al. [13] demonstrated that Ubqln4 negatively interacts with RNF114 to 
inhibit the proliferation of tumor cells. Gopeshwar et al. [14] identified that RNF114 is upregulated by DNA copy number increases and is a potential oncogene in cervical cancer. There are an increasing number of reports regarding RNF114 in tumors, whereas detailed reports regarding its role in GC are lacking.

EGR1, a transcription factor, plays a dual role in tumor progression by regulating downstream target genes. EGR1 may suppress tumor effects in breast tumors [15], rhabdomyosarcoma [16] and bladder tumors [17], while promoting tumor effects in prostate cancer [18] and lung cancer [19]. Surprisingly, contrary results have been reported for the role of EGR1 in gastric cancer [20,21], the mechanism of which is still unclear. MicroRNAs (miRNAs), 22 nucleotides on average, are a group of evolutionarily conserved single-stranded non-coding RNAs. miR-218-5 $p$ has been implicated in various types of cancer [22]. As a tumor suppressor, miRNAs have been found to be downregulated in GC. Tianqi suggested that the long non-coding RNA SNHG12 promotes GC metastasis by regulating the miR-218-5p/YWHAZ axis [23], while Min found that miR-218 suppresses gastric cancer cell cycle progression through the CDK6/Cyclin D1/E2F1 axis [24].

In this study, we analyzed the characteristics of variant RNF114 mRNA levels in patients with GC. These results demonstrated that RNF114 was highly expressed at the mRNA or protein level in GC, and that patient prognosis was more reliant on upregulated RNF114 than downregulated levels. In addition, miR-218-5p is a potential regulator that controls RNF114 expression, to some extent, and can mediate EGR1 degradation via the ubiquitylation approach.

\section{Materials and methods}

\section{Bioinformatic analysis}

The differential expression of RNF114 across various cancers was investigated using TIMER (https://cistrome.shinyapps.io/timer/) [25], GEPIA (Gene Expression Profiling Analysis) (http:// gepia.cancer-pku.cn/) [26], and Oncomine databases (https://www.oncomine.org/) were used to analyze RNF114 expression in GC. RNA-seq expression profiles of GC were downloaded from The Cancer Gneome Atlas (TCGA) database (https://portal.gdc .cancer.gov/) and processed using R software. These files were composed of sample expression files and phenotypic classification files (Format: .gct and .cls). We analyzed these datasets for signaling pathways of the RNF114 gene using GSEA software 4.0.3. The correlation between RNF114 mRNA expression and overall survival (OS) in GC was analyzed using the Kaplan-Meier plotter (http://kmplot.com/) [27], which is the most comprehensive survival website. DNA methylation of RNF114 was analyzed using MethSurv (https://biit.cs.ut.ee/methsurv/) [28], which is an interactive web portal that provides survival analysis based on DNA methylation biomarkers using TCGA data. Potential miRNAs and transcription factors associated with RNF114 were predicted using NetworkAnalysis3.0 (https://www. networkanalyst.ca/) [29], a comprehensive network visual analytics platform for gene expression analysis. Potential miRNAs were obtained and the co-expression relationship between potential miRNAs and targeted genes was analyzed using the starBase v2.0 (http://starbase.sysu.edu.cn/) [30], which mainly focused on miRNA-target interactions.

\section{Patient data and tissue samples}

We collected 19 fresh GC tissues and peritumoral normal gastric mucosa at the Department of General Gastrointestinal Surgery of the First Affiliated Hospital of Nanchang University in 2018. Paraffinembedded GC samples $(n=123)$ and normal gastric gland samples $(n=20)$ were obtained from the Department of Pathology of the First Affiliated Hospital of Nanchang University.

\section{Quantitative real time PCR (qRT-PCR) and western blotting}

The qRT-PCR experiments were performed according to the manufacturer's protocol and according to a previous study $[31,32]$. The HACTB gene was used as an endogenous control. HACTB: forward 5'-CATCCGCAAAGACCTGTACG-3'; reverse 5'-CCTGCTTGCTGATCCACATC-3', RNF114: forward 5'-GGGAGACCCCAACTACCG-3'; reverse 5'-AGCGCTGCAACACCTGAT-3'. Western blotting was performed according to the manufacturer's protocol, as described in a previous study [33]. The following antibodies were used in this study: $\beta$-actin (1:1000 dilution, Affinity, China) and RNF114 (1:700 dilution, Altas, Sweden).

\section{Immunohistochemistry (IHC) and evaluation}

Paraffin-embedded samples were sliced into 3.5 $\mu \mathrm{m}$ thick sections, which were coated with rabbit anti-RNF114 antibody (1:200 dilution) after deparaffinization, rehydration, and antigen repair. Two independent pathologists evaluated the stained slides using a microscope (Nikon, Tokyo, Japan). Each case was scored based on the following criteria: intensity $(0$, negative; 1 , weak $(+) ; 2$, moderate $(++) ; 3$, strong $(+++))$ and percentage of positive staining, area (0: $1-25 \%$; 2: $26-50 \%$; 3: $51-75 \%$; 4: 76-100\%). The ultimate score was the product of the intensity and 
area (0-12). Samples with a score $\geq 6$ were defined as having high RNF114 expression, while samples with a score $<6$ were regarded as having low RNF114 expression.

\section{Cell culture, infection, and siRNAs}

All GC cell lines, including HGC-27, MKN-45, MGC-803, SGC-7901, BGC-823, AGS, and the normal gastric epithelial cell line (GES-1), were maintained in RPMI 1640 medium with $10 \%$ fetal bovine serum (HyClone GE Healthcare Life Sciences, Logan, UT, USA) at $37^{\circ} \mathrm{C}$ in a $5 \% \mathrm{CO}_{2}$ atmosphere.

We obtained three sequences of RNF114 small interfering RNA (Si-1: sense 5'-GCCACCAUUAAGG AUGCAUTT-3', antisense 5'-AUGCAUCCUUAAUG GUGGCTT-3' Si-2, sense 5'-GUGGAACACUGCAAA UUAUTT-3', antisense 5'- AUAAUUUGCAGUGUUC CACTT-3'; Si-3: sense 5'-GUGGCUACUUGUUCCAA AUTT-3', antisense 5'-AUUUGGAACAAGUAGCCA CTT-3') and a negative control RNA from GenePharma Company (Shanghai, China). These siRNAs and negative control RNA were transfected into HGC-27 and BGC-823 cells.

The lentivirus vector included a short hairpin RNA of knockdown RNF114 expression (shRNA) and a negative control sequence (shNC) designed by GenePharma Company. We used shRNF114 (5'-GTG GCTACTTGTTCCAAAT-3') and shNC (5'-TTCTCCG AACGTGTCACGT-3') to infect HGC-27 and BGC-823 cells with polybrene $(5 \mu \mathrm{g} / \mathrm{mL})$. We tested transfection efficiency and selected using puromycin for subsequent cell functional experiments.

\section{Immunofluorescence (IF)}

Tissue IF and cell IF were performed as described previously [34]. Primary antibodies against RNF114 (1:100 dilution) were used in this study. Samples stained for RNF114 were incubated with an Alexa Fluor 594-conjugated goat anti-rabbit secondary antibody (Elabscience, China), and the nuclear samples were stained with DAPI.

\section{Cell function assays}

\section{5-Ethynyl-2'-deoxyuridine (EdU) assay}

Lentivirus-transfected GC cells were cultured in 96-well plates. First, $200 \mu \mathrm{L}$ of a $50 \mu \mathrm{mol} / \mathrm{L}$ EdU solution (Guangzhou RiboBio Co.,LTD, China) was added to the cultured cells. After washing, fixation, and permeabilization, $100 \mu \mathrm{L}$ of $1 \times$ Apollo solution was added to the cells. Cellular DNA was then stained with $100 \mu \mathrm{L}$ of $1 \times$ Hoechst 33342 solution. A fluorescence microscope was used to capture images of EdU- and Hoechst 33342-positive cells.

\section{Colony formation}

The cells were seeded in 6-well plates and cultured at $37{ }^{\circ} \mathrm{C}$ in a $5 \% \mathrm{CO}_{2}$ atmosphere for $10-14$ days. Colonies were fixed with $4 \%$ paraformaldehyde, stained with $0.5 \%$ crystal violet solution, and washed before counting.

\section{Transwell assay}

Transwell chambers with $8 \mu \mathrm{m}$ pores with or without Matrigel (BD Biosciences) were used for invasion and migration assays, as described in a previous study [35]. The chamber was fixed with paraformaldehyde, stained with crystal violet, and observed under a microscope.

\section{Scratch-wound assay}

The cells were then placed in sterile 6-well plates. A "wound" was scratched using a sterile $10 \mu \mathrm{L}$ pipette tip when cells were grown to $80-100 \%$ confluence. Images were obtained using a microscope at 0 and $24 \mathrm{~h}$, and analyzed.

\section{Methylation-specific PCR (MSP)}

Genomic DNA was isolated from cells samples by using columnar centrifugation, and was treated with sodium bisulfite. PCR was performed using methylated and unmethylated primers, respectively. methylated primers: forward 5'- AGTGTAAGTGGTG TCGAATTAACGAT -3'; reverse 5'- AAACTAAC GAATAAACCGCCCCT-3', unmethylated primers: forward 5'- AGTGTAAGTGGTGTTGAATTAATGAT AAA -3'; reverse 5'- CAAAACTAACAAATAAACC ACCCCTC $-3^{\prime}$. PCR products were identified with agarose gel electrophoresis.

\section{In vivo tumor xenograft model}

Ten mice were purchased from SJA Laboratory Animal Co., Ltd (Hunan, China) and randomly separated into shNC and shRNA groups. A total of 1 $\times 10^{6}$ cultured shNC-BGC-823 and shRNF114-BGC823 cells were diluted with PBS and injected into the right thigh of each nude mouse. After 1 week, subcutaneous xenograft tumors were observed, and the tumor size and body weight were measured every week. Five weeks later, the tumors in the right thigh of nude mice were resected subtly after sacrifice, and their weights and sizes were measured. All procedures in this study were approved by the Institutional Animal Care Committee of the First Affiliated Hospital of Nanchang University.

\section{Statistical analysis}

Data were processed using SPSS version 19.0 (SPSS, Chicago, USA) and GraphPad Prism 7.0 software (GraphPad Software, La Jolla, CA, USA). Student's $t$-test or one-way analysis of variance was 
used to analyze differences between groups. The chi-square test and Fisher's exact test were used to compare the relationship between RNF114 expression and clinicopathology. Kaplan-Meier curves and Cox regression models were used to analyze survival rates. All data are expressed as means $\pm \mathrm{SD}$, and $P$ values $<0.05$ were considered statistically significant.

\section{Results}

\section{RNF114 overexpression was observed in GC using bioinformatics analysis}

To explore RNF114 mRNA expression among different tumors, TCGA datasets associated with tumors were extracted and calculated using the TIMER server. As shown in Figure 1A, RNF114 mRNA was notably overexpressed in most lethal tumors, particularly in GC. Meanwhile, pair-differed analysis indicated that RNF114 expression was markedly higher in the tumor than in the normal group (Figure 1B). Combined with the GTEx and TCGA GC datasets, increasing the normal sample, RNF114 mRNA expression was highly expressed in GC compared with normal samples (Figure 1C). Chen data, extracted from the Oncomine database, revealed similar outcomes regarding RNF114 expression (Figure 1D). The bioinformatics results suggested that RNF114 mRNA was overexpressed in GC, and this finding requires further study.

\section{RNF 114 was elevated in GC tissues and poor outcome was observed with high RNF114- expression patients}

To investigate the correlation between RNF114 expression and clinicopathological characteristics in GC patients, we performed immunohistochemistry (IHC) and evaluated 20 normal gastric tissues and 123 primary GC tissues. The IHC results revealed that
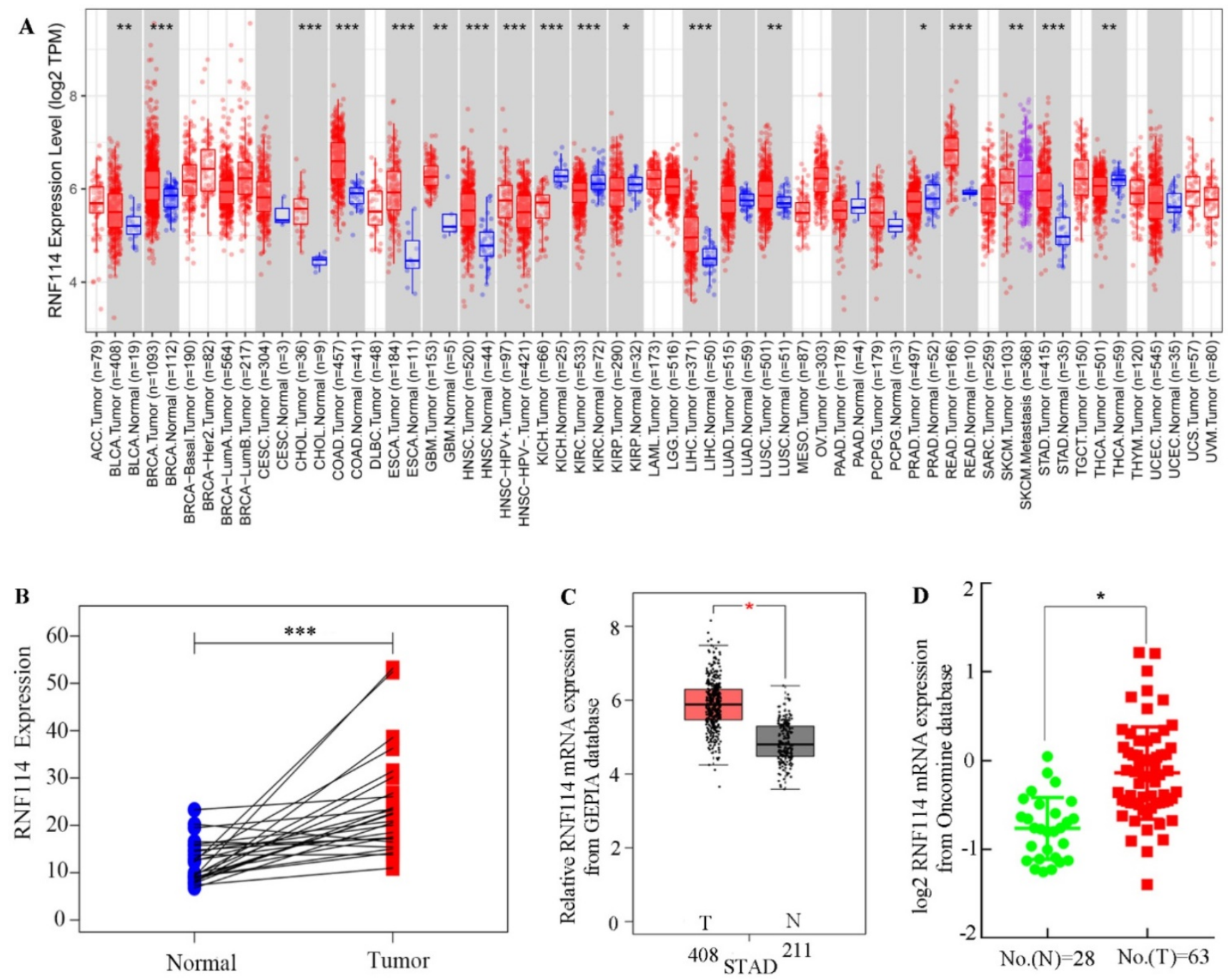

Figure 1. RNF114 was overexpressed in gastric cancer. (A) The different expressions of RNFI I4 mRNA among pan-cancer from TIMER database. (B) Differences in RNFI I 4 expression for GC were analyzed using a paired $t$-test analysis. (C) RNF114 was overexpressed in GC according to the GEPIA database. (D) RNF114 was overexpressed in GC with Chen sample data according to the Oncomine database $(* * * p \leq 0.001, * * p \leq 0.01, * p \leq 0.05)$. 
RNF114 expression was higher in tumor tissues than in adjacent normal tissues (Figure 2A). According to whether the coloring score of RNF114 protein was greater than or less than 6 points, all patients were divided into high expression and low expression groups. As described in Table 1, RNF114 protein expression was closely associated with tumor localization $(p<0.001)$, T stage $(p=0.003)$, and lymph node metastasis $(p=0.001)$; however, RNF114 expression was independent of other parameters, including age, gender, tumor diameter, tumor differentiation, cancer nodule number, and vascular invasion. Compared with the low expression group, the high expression group had a worse overall survival (OS) $(p<0.001)$, observed using Kaplan-Meier analysis (Figure 2B), which was also confirmed by the K-M Plotter database of Affymetrix gene chip ID: 200868_s_at (Figure 2C). Univariate and multivariate analyses showed that RNF114 levels (HR=1.772, $p=0.015$ ) were independent prognostic factors in patients with GC (Table 2).

Table 1. Association between the expression of RNF114 and clinicopathological characteristics of patients with GC

\begin{tabular}{|c|c|c|c|c|c|}
\hline $\begin{array}{l}\text { Clinicopathological } \\
\text { characteristics }\end{array}$ & $\mathrm{n}$ & $\begin{array}{l}\text { Low } \\
\text { expression }\end{array}$ & $\begin{array}{l}\text { High } \\
\text { expression }\end{array}$ & $\chi^{2}$ & P-value \\
\hline Total & 123 & 57 & 66 & & \\
\hline Gender & & & & 1.665 & 0.197 \\
\hline Male & 88 & 44 & 44 & & \\
\hline Female & 35 & 13 & 22 & & \\
\hline Age (y) & & & & 0.109 & 0.741 \\
\hline$<60$ & 71 & 32 & 39 & & \\
\hline$\geq 60$ & 52 & 25 & 27 & & \\
\hline Tumor localization & & & & 12.139 & $0.000^{*}$ \\
\hline Down & 92 & 51 & 41 & & \\
\hline $\mathrm{Up}$ & 31 & 6 & 25 & & \\
\hline Tumor diameter $(\mathrm{cm})$ & & & & 2.928 & 0.087 \\
\hline$<4$ & 61 & 33 & 28 & & \\
\hline$\geq 4$ & 62 & 24 & 38 & & \\
\hline Tumor differentiation & & & & 0.875 & 0.350 \\
\hline Low & 85 & 37 & 48 & & \\
\hline Middle + High & 38 & 20 & 18 & & \\
\hline T stage & & & & 8.734 & 0.003 \\
\hline $\mathrm{T} 1+\mathrm{T} 2$ & 32 & 22 & 10 & & \\
\hline $\mathrm{T} 3+\mathrm{T} 4$ & 91 & 35 & 56 & & \\
\hline Lymph node metastasis & & & & 12.032 & 0.001 \\
\hline no & 39 & 27 & 12 & & \\
\hline yes & 84 & 30 & 54 & & \\
\hline Cancer nodule number & & & & 3.037 & $0.104^{*}$ \\
\hline no & 113 & 55 & 58 & & \\
\hline yes & 10 & 2 & 8 & & \\
\hline Vascular invasion & & & & 2.603 & 0.107 \\
\hline no & 77 & 40 & 37 & & \\
\hline yes & 46 & 17 & 29 & & \\
\hline
\end{tabular}

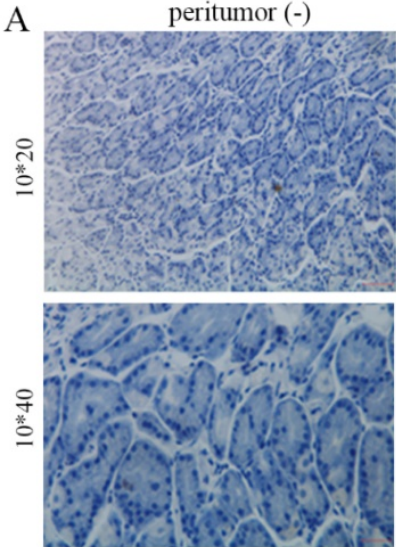

B

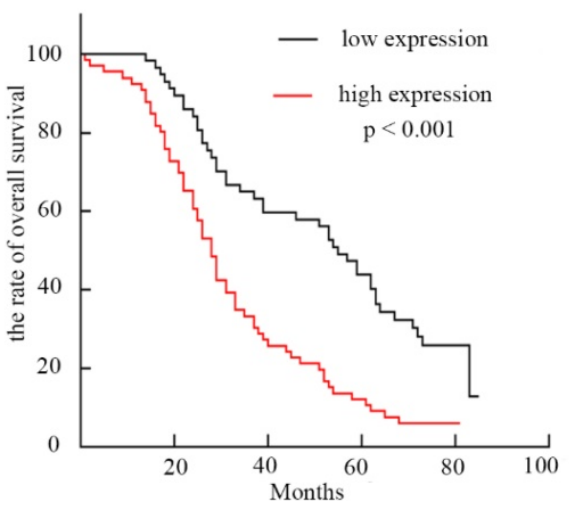

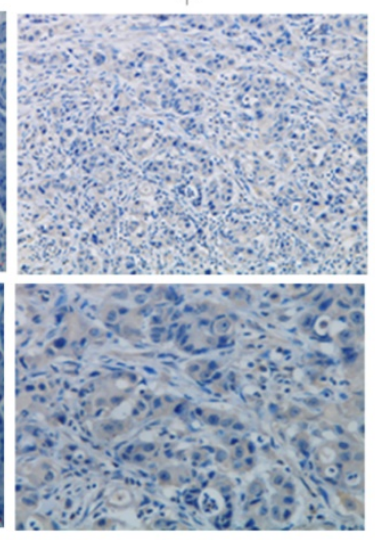
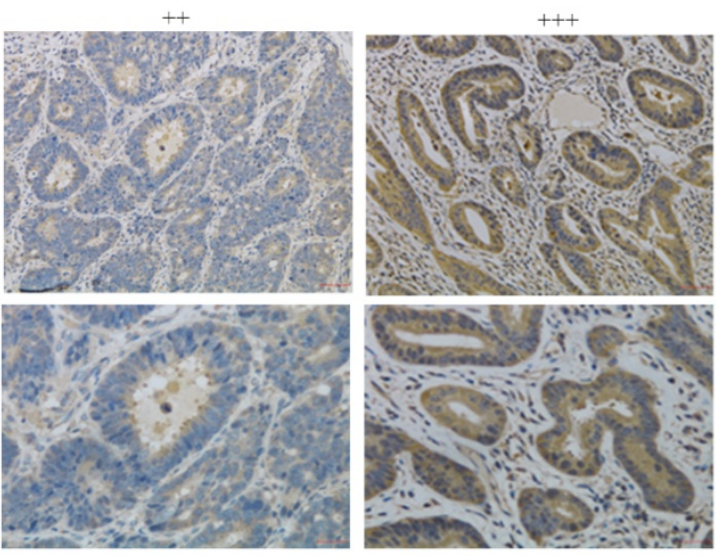

C

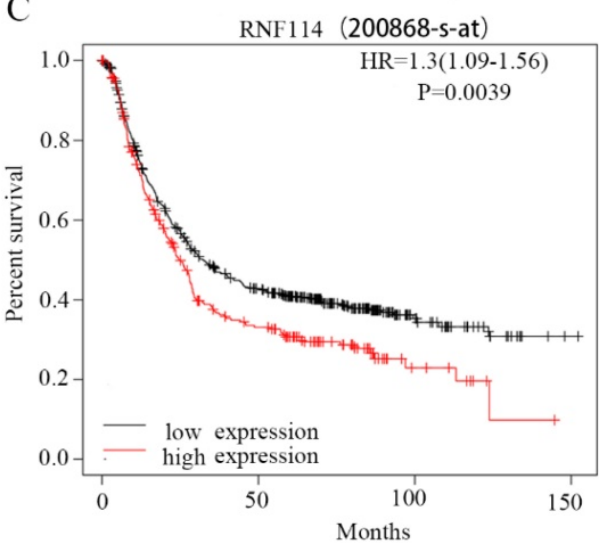

Figure 2. Analysis of survival with GC based on RNF114 expression via IHC. (A) Representative images of RNF114 staining in GC and adjacent normal tissues (+: weak, ++: moderate, +++: strong, $10 * 20$ and $10 * 40$ respectively represents an image magnified 200 times and 400 times). (B) Kaplan-Meier overall survival analysis of 123 patients stratified by RNF114 high and low expression levels. (C) The Kaplan-Meier Plotter database also showed that high expression of RNF114 correlated with shorter OS. 


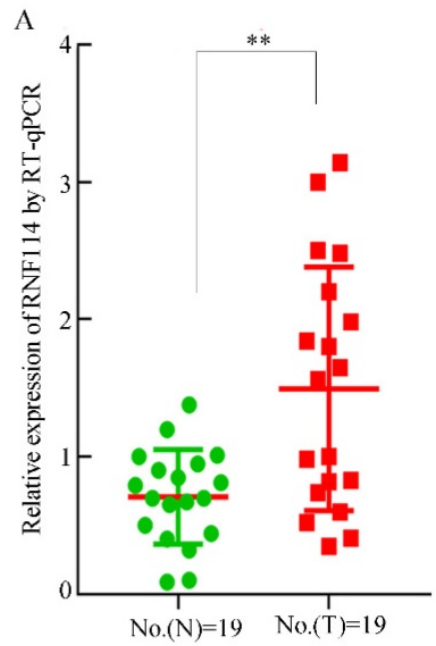

D

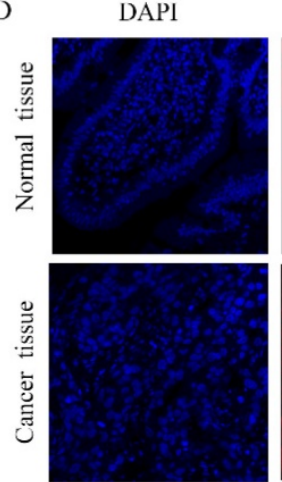

RNF 114

$\beta$-actin
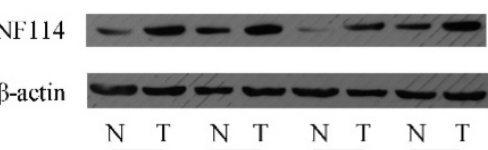
$\overline{\text { case1 }} \overline{\text { case2 }} \overline{\text { case3 }} \overline{\text { case4 }}$
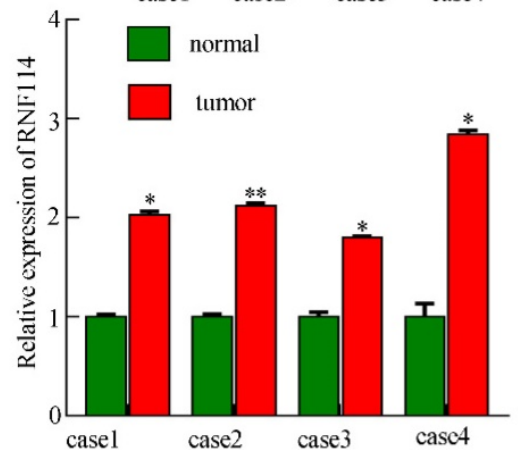

$\mathrm{RNF} 114$
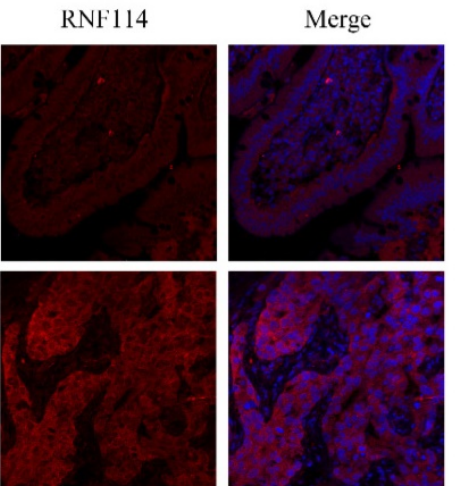

E

DAPI
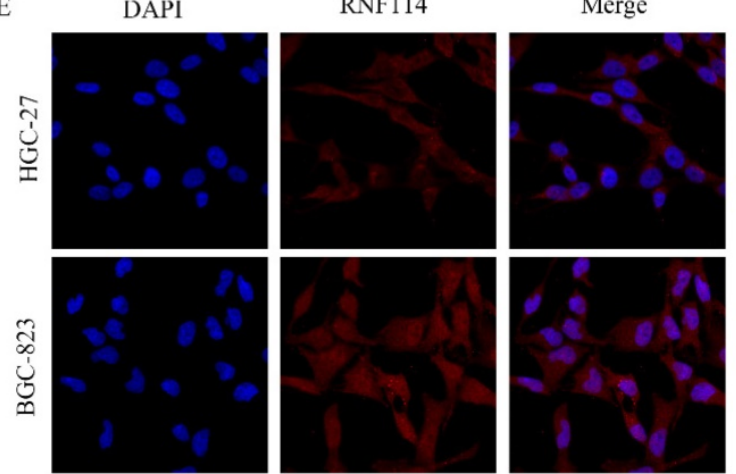

Figure 3. RNFI 14 was overexpressed in GC tissues and cells. (A-C) RNF1 14 expression levels were high in GC tissues, as observed using qRT-PCR and western blotting. $A$ indicates qRT-PCR results with fresh tissues, $B$ indicates western blot results with fresh tissues, and $C$ indicates western blotting results with cells. (D-E) The expression and localization of RNF114 were examined using immunofluorescence staining in tissues (Figure D) and BGC-823 cells (Figure E) (*** $p \leq 0.001, * * p \leq 0.01$, * $p \leq 0.05$ ).

To assess RNF114 expression in tissues and cells from RNA sequences and proteins, we detected RNF114 mRNA and protein levels in 19 pairs of fresh GC tissues, contiguous normal tissues, and GC cells. As shown in Figure 3A, higher mRNA levels of RNF114 were observed in GC tissues using qRT-PCR. Figure $3 \mathrm{~B}$ shows a representative western blotting result from 19 pairs of fresh samples, and the expression of RNF114 protein in GC tissues was higher than that in the adjacent tissues. As shown in Figure 4C, RNF114 levels were lower in GES-1 cell lines than in GC cell lines, as determined by western blotting. Additionally, HGC-27 and BGC-823 cells showed higher expression than other cells. Subsequently, we performed IF staining of the GC tissues and cells. DAPI was used to stain the cellular nuclei (blue fluorescence), and the RNF114 protein was stained with a fluorescent antibody (red fluorescence). Red fluorescence (RNF114 protein staining) levels were lower in normal tissues than in cancer tissues (Figure 3D). In the two cell lines, RNF114 was mainly located in the cytoplasm and nuclei (Figure 3E). Based on the investigation of RNF114, it was elevated in GC tissues and poor

outcome with high RNF114-expression patients.

Table 2. Univariate and multivariate analysis of the association between prognosis, clinicopathological characteristics, and RNF1 14 expression in GC

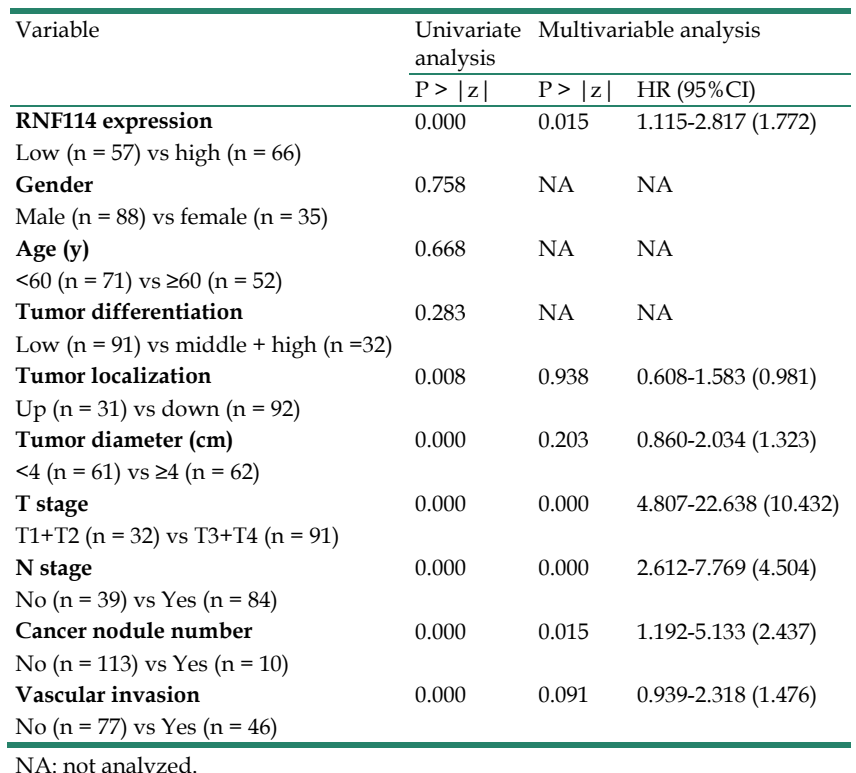


$\mathbf{A}$

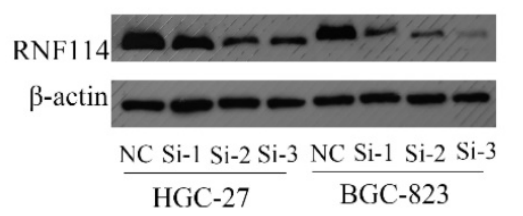

B
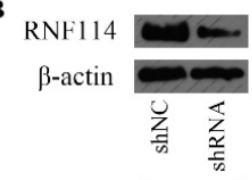
HGC-27

HGC-27

D

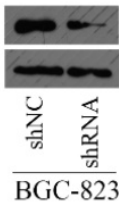

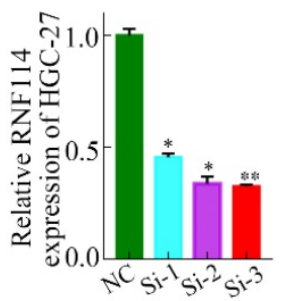

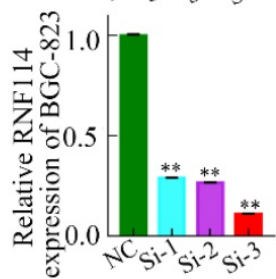

BGC- 823
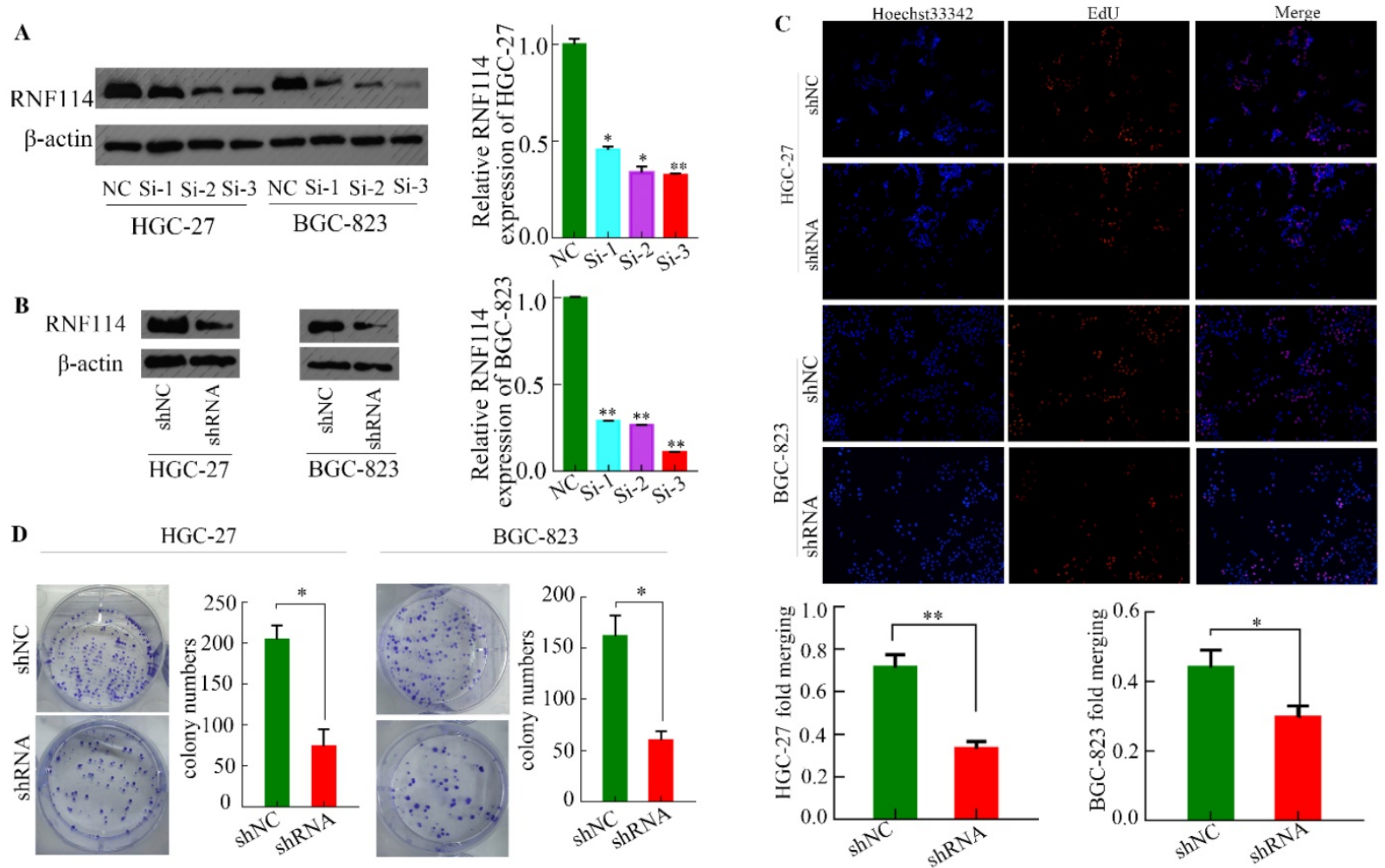

E

HGC27
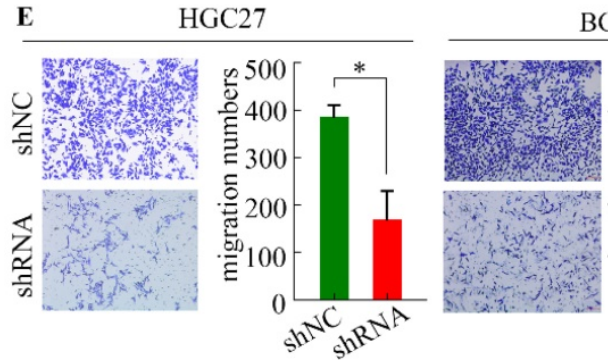

BGC-823

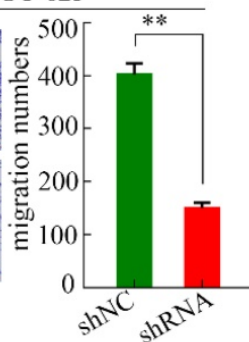

HGC-27

BGC-823

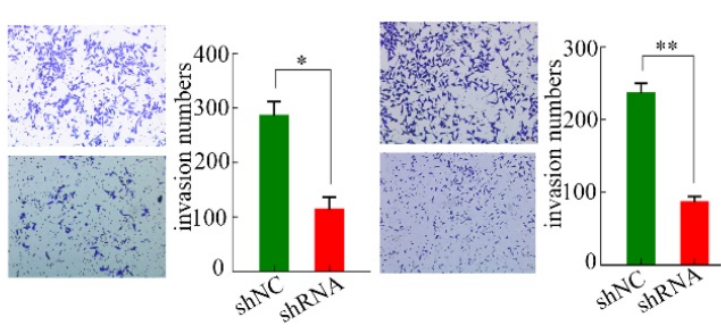

G
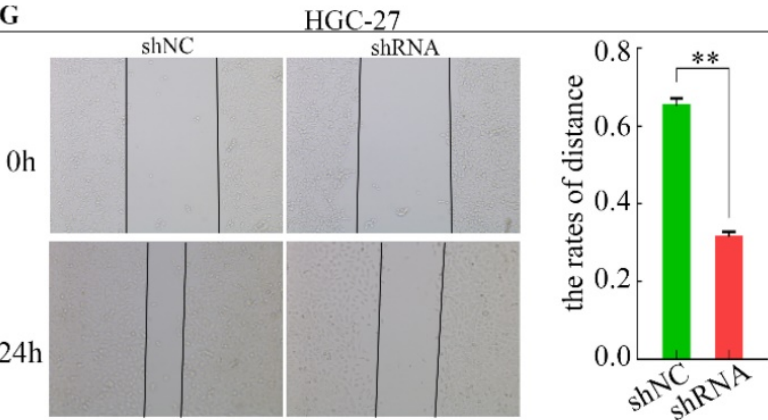

H
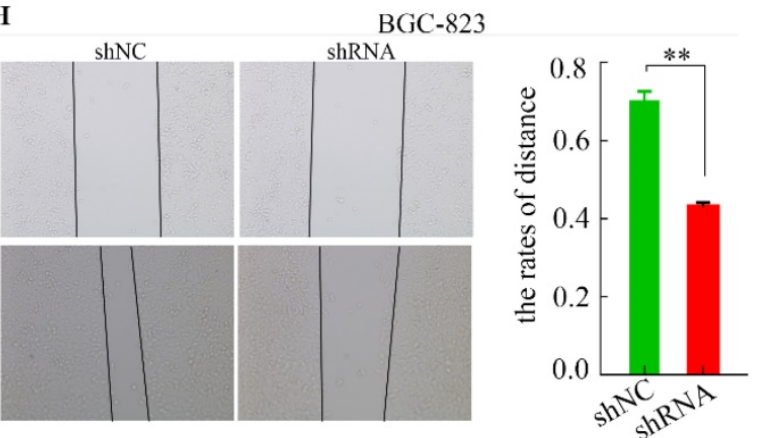

Figure 4. RNFI14 knockdown inhibits GC cell proliferation and invasion. (A) The expression of RNF114 was downregulated by siRNA in HGC-27 and BGC-823 cells. (B) The expression of RNF114 was downregulated by shRNA in HGC-27 and BGC-823 cells. (C) The proliferation of cells with RNFI I 4 knockdown was analyzed using EdU assays. (D) Colony formation assays were used to detect the effect of RNF114 on cell growth. (E-F) Migration and invasion abilities of RNF114 knockdown cells were investigated using Transwell assays. E) Migration assays, F) invasion assays, and (G-H) scratch-wound assays were used to assess healing ability inHGC-27 cells (G) and BGC-823 cells $(\mathrm{H})\left(* * * \leq 0.001, * * p \leq 0.01,{ }^{*} p \leq 0.05\right)$.

\section{RNFI 14 silencing decreased GC cell line proliferation, migration, and invasion}

We designed three siRNAs (Si-1, Si-2, and Si-3) targeting RNF114 and an NC sequence to interfere with the expression of RNF114 in HGC-27 and
BGC-823 cell lines. As shown in Figure 4A, all siRNAs exerted interference effects, with siRNA 3 showing the best effect. Thus, we selected Si-3 siRNA sequence for lentivirus packaging and subsequent infection of the two cell lines to constructe stable RNF114knockdowned cells (Figure 4B). 

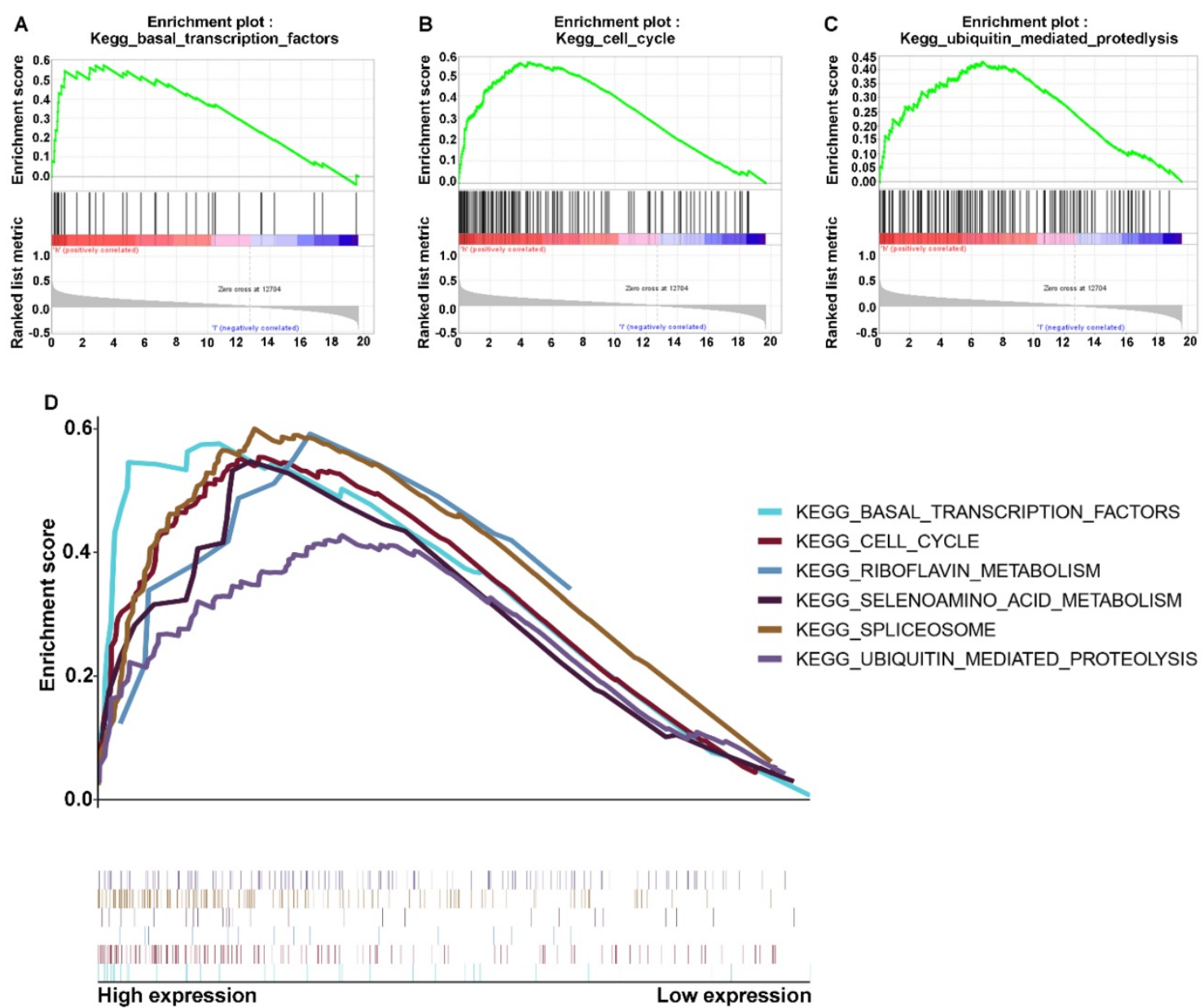

Figure 5. Enriched signaling pathways associated with RNFII4 via GSEA. (A-C) The signaling pathway genes were enriched in RNFI 44 high expression. A indicates the ubiquitin-mediated proteolysis signaling pathway. B indicates the basal transcription factors signaling pathway. C indicates the cell cycle signaling pathway. (D) Multiple signaling pathways enriched in high RNFI l 4 gene expression.

EdU and colony formation experiments were performed to detect the proliferation ability of cells infected with the lentivirus. As shown in Figure 4C, the nuclei were stained with Hoechst 33342 (blue fluorescence), and the amplified DNA was stained with Apollo solution (red fluorescence). Using "ImageJ" software to scan the EdU-associated images, we found that approximately $36.95 \%$ of cells were positive for EdU in the shRNA group of HGC-27 cells, while approximately $77.42 \%$ was seen in the shNC group. Similarly, in the BGC-823 cell line, the proportion was approximately $42.21 \%$ in the shNC group and $29.73 \%$ in the shRNA group. Immediately after, the colony formation experiment showed similar trends (Figure 4D). To some extent, the cell proliferation ability of GC was weakened by RNF114 silencing.

To identify the effect of RNF114 on GC migration and invasion, we performed transwell and scratchwound assays. The total number of migrated cells was lower in the shRNA group than in the shNC group (Figure 4E). The invasion ability was greater in the shNC group than in the shRNA group (Figure 4F).
The control group healed faster in the scratch-wound assay than the RNF114-knockdown group for both HGC-27 and BGC-823 cell lines (Figure 4G-H).

\section{Signaling pathway with GSEA and the regulation network of RNFII4}

The above results indicated that RNF114 may be associated with proliferation and metastasis in GC, but its detailed regulatory mechanism remains elusive. To explore the involvement of RNF114 in the signaling pathway, we assessed the co-expression between differentially associated gene expression with signaling and in two subtypes, which were divided by the expression of RNF114. We performed gene set enrichment analysis (GSEA), and the results showed that the malignant hallmarks of tumors, including ubiquitin-mediated proteolysis (normalized enrichment score [NES] $=1.62, \quad p<0.031)$, basal transcription factors (NES=1.81, $p<0.001$ ), and cell cycle signaling (NES=1.85, $p=0.017)$, were dynamically correlated with the high RNF114 subtype (Figure 5A-C). In addition, the significant pathways with RNF114 expression in GC data also included 
spliceosome, selenoamino acid metabolism, and riboflavin metabolism, as shown in Figure 5D. Hence, binding RNF114 structure attributes, the ubiquitinmediated proteolysis-signaling pathway may be implicated in the regulation of pivotal factors.

To determine the molecular mechanism of RNF114, we analyzed the network of transcription factors and microRNAs using NetworkAnalyst3.0, a comprehensive network visual analytics platform for gene expression analysis (Figure 6A). There were four transcription factors and microRNA closely correlated with RNF114, respectively, which were SP1, MAX, EGR1, and USF1, and hsa-miR-492, hsa-miR-506, hsa-miR-218, and hsa-miR-124. Using the starBase databases, we found that hsa-miR-218-5p was associated with RNF14, the presence of an RNA-binding site is displayed in Figure 6B, and the differing and corresponding expression of
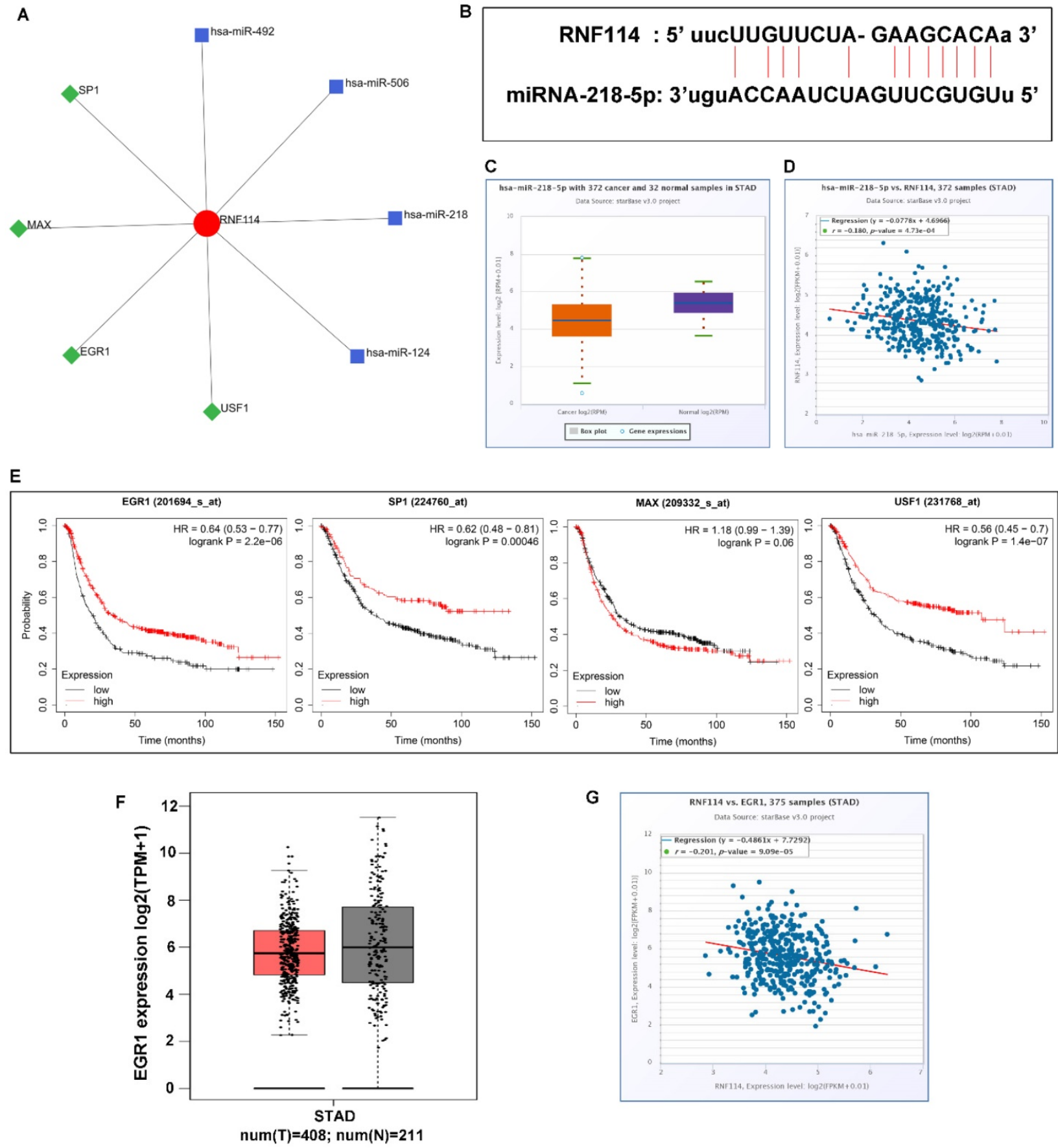

Figure 6. Potential miRNA and transcription factor co-expression network in GC. (A) miRNAs and transcription factors that interacted with RNF114 predicted by the NetworkAnalyst3.0 (B) The potential sequences of miR-218-5p binding sites at the 3' UTR of RNFI 14. (C) miR-218-5p differential expression in GC and normal tissue from the starBase database. (D) Negative correlation between miR-218-5p and RNFI I 4 expression. (E) Result of EGR1, SPI, MAX, and USF1 prognostic analysis for GC in the Kaplan-Meier plotter databases. (F) EGRI differential expression in GC and normal tissue from the GEPIA database (red represent tumor, grey represent normal). (G) The negative correlation between miR-218-5p and RNF114 expression from the starBase database. 
hsa-miR-218-5p was evident in GC (Figure 6C-D). Down-expression of hsa-miR-218-5p may attenuate regulation and release RNF114 expression in GC. The association between transcription factors related to RNF114 and patient outcomes was analyzed using the Kaplan-Meier plotter database. The low expression of SP1, EGR1, and USF1 was associated with poor overall survival, while MAX expression was not significantly associated with the overall survival of patients with GC (Figure 6E). The trend of lower EGR1 expression in GC than in normal GC was analyzed using GEPIA from TCGA and GTEx databases (Figure 6F). Additionally, EGR1 expression

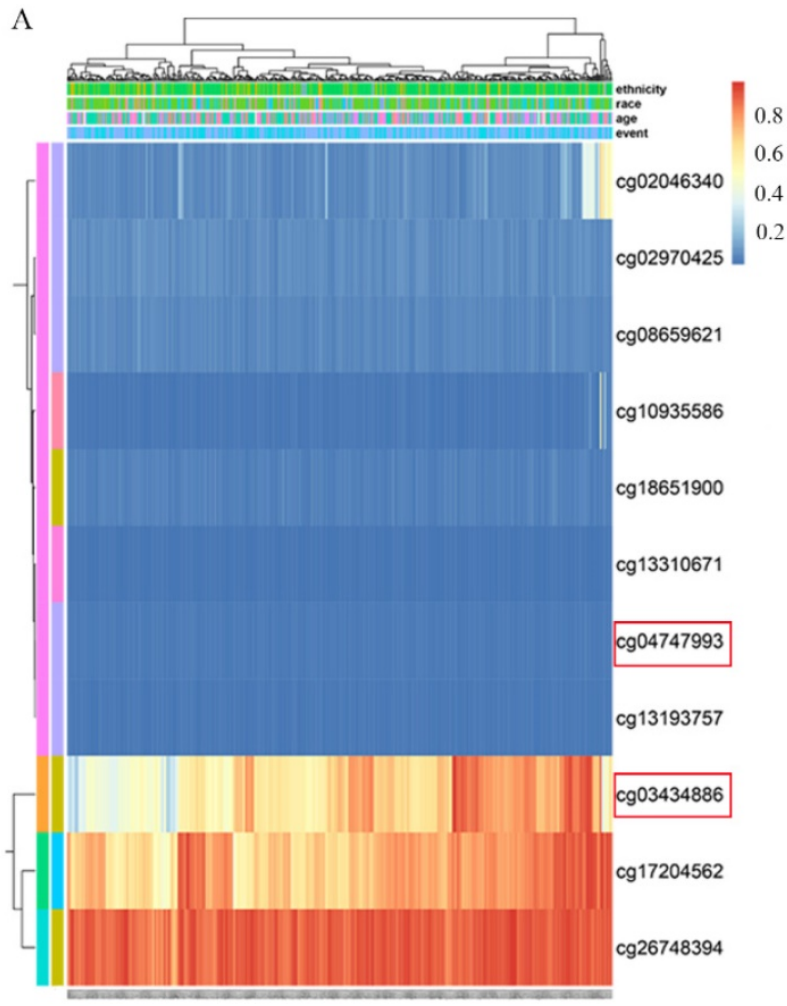

D

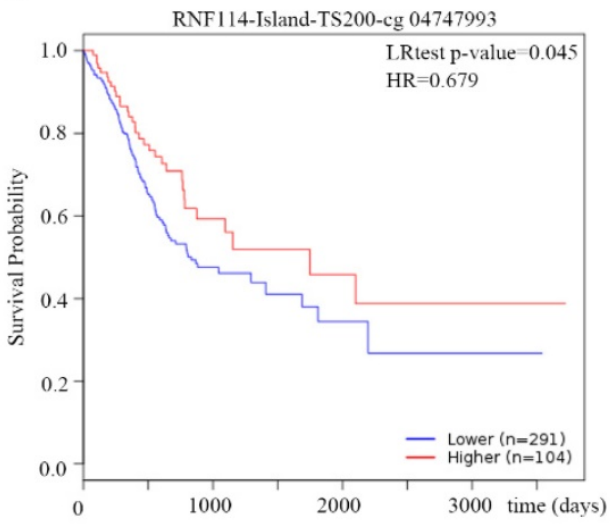

was negatively correlated with RNF114, as concluded from the starBase database (Figure 6G). Based on these findings, we suggest that RNF114 may trigger the ubiquitylation degradation of EGR1 and indirectly contribute to GC development.

\section{RNFI 4 methylation in GC}

DNA methylation of RNF114 was analyzed using the MethSurv network tool from the GC TCGA database. All methylation probes associated with RNF114 are shown in Figure 7A. The red and blue heatmaps represent hypermethylation and hypomethylation, respectively, in GC samples.
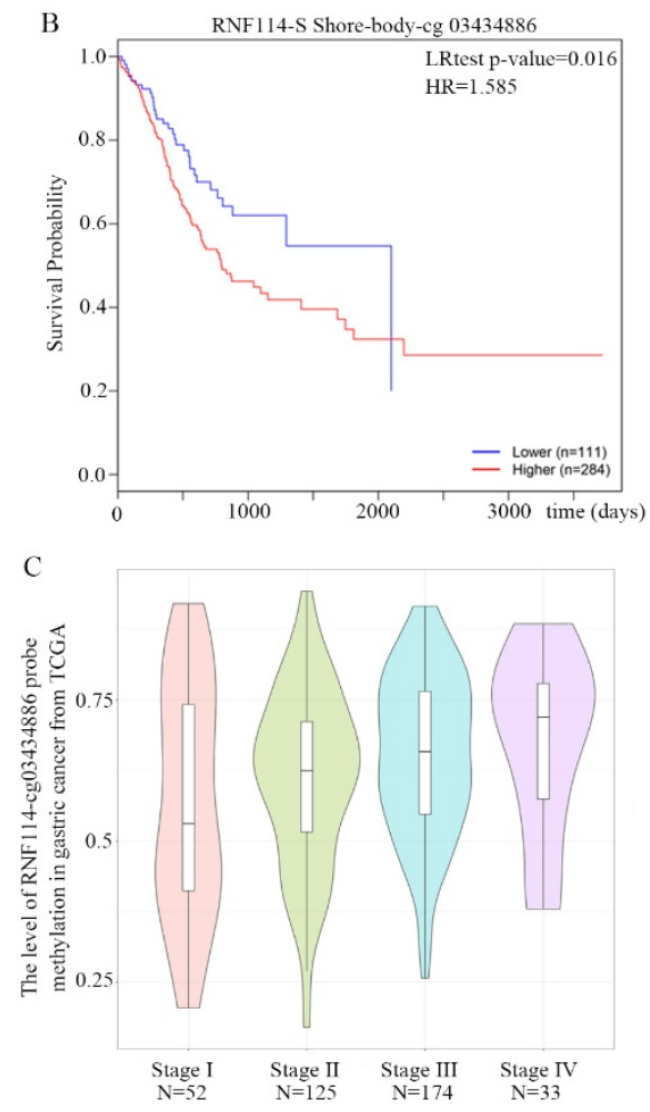

E

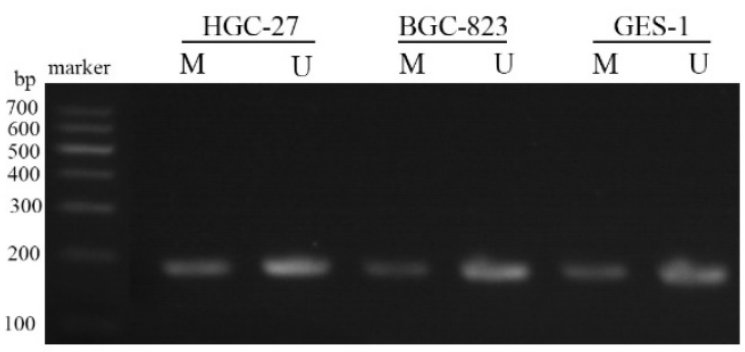

Figure 7. DNA methylation of RNFII4 in GC from MethSurv network tool. (A) The cluster of RNFII4 methylation in MethSurv network tool. Red to blue: hypermethylation to hypoexpression. (B) The Prognostic Values of methylation-associated probes cg03434886 with RNFI I 4. (C) The relationship between RNF1 14-associated probe cg03434886 and tumor stage. (D) The Prognostic Values of methylation-associated probes cg4747933 with RNFI I4. (E) MSP detected the RNFI I 4 methylation in gastric cancer cells ( $M$ and $U$ present methylated and unmethylated respectively). 
Cg03434886 probes detected the RNF114 gene in the hypermethylation region, and hypermethylation had a worse outcome than hypomethylation (Figure 7B). Further analysis revealed that the level of methylation was significantly associated with tumor staging, and the later the tumor staging, the higher the methylation (Figure 7C). This result revealed that RNF114cg03434886 hypermethylation may stimulate RNF114 expression in GC. However, RNF114-cg04747993 was less modified with methyl in GC chip results and the GC patient with RNF114-cg04747933 hypermethylation has a better prognosis than hypomethylation (Figure 7D). The methylation status of RNF114 in the HGC-27, BGC-823 and GES-1 cell lines was detected using MSP. The bands in Figure 7E indicated RNF114 partially methylated state, while more unmethylated RNF114 in gastric cells. In general, gene methylation will lead to the inhibition of gene expression, nonetheless, RNF114 has a controversial result, which is a very interesting phenomenon worthy of further study.

\section{RNFII4 silencing suppressed xenograft tumor growth}

To evaluate the influence of RNF114 in vivo, a xenograft tumor mouse model was constructed. As shown in Figure 8A-B, knockdown of RNF114 inhibited tumor growth in the nude mice. The tumor volumes in the shRNA group were significantly lower

than those in the shNC group (Figure 8C). Similarly, we clearly showed that the tumor weights were higher in the shNC group than in the shRNA group (Figure 8D).

\section{Discussion}

RNF114 was first reported as a novel susceptibility gene for psoriasis, while several studies have demonstrated that RNF114 plays a critical role in tumor development [36,37]. Genetic and environmental changes play a decisive role in GC formation. It is well known that geographical environment, diet and lifestyle factors, smoking, and Helicobacter pylori infection are high-risk GC factors [38-40], and changes in these factors can cause somatic gene mutations promoting GC, such as HER2, VEGF, and RAS [41-43]. According to existing research, there is a lack of sufficient studies to elucidate the relationship between RNF114 and GC. We first studied and reported the relationship between RNF114 and GC in detail. As shown in Figure 1, using bioinformatics analysis, the expression of RNF114 in GC was significantly higher than that in normal tissues. We verified these results using qRT-PCR and western blotting (Figures 2 and 3). Through cell function experiments, we found that silencing RNF114 affected the biological behavior of GC cells, such as proliferation and invasion.
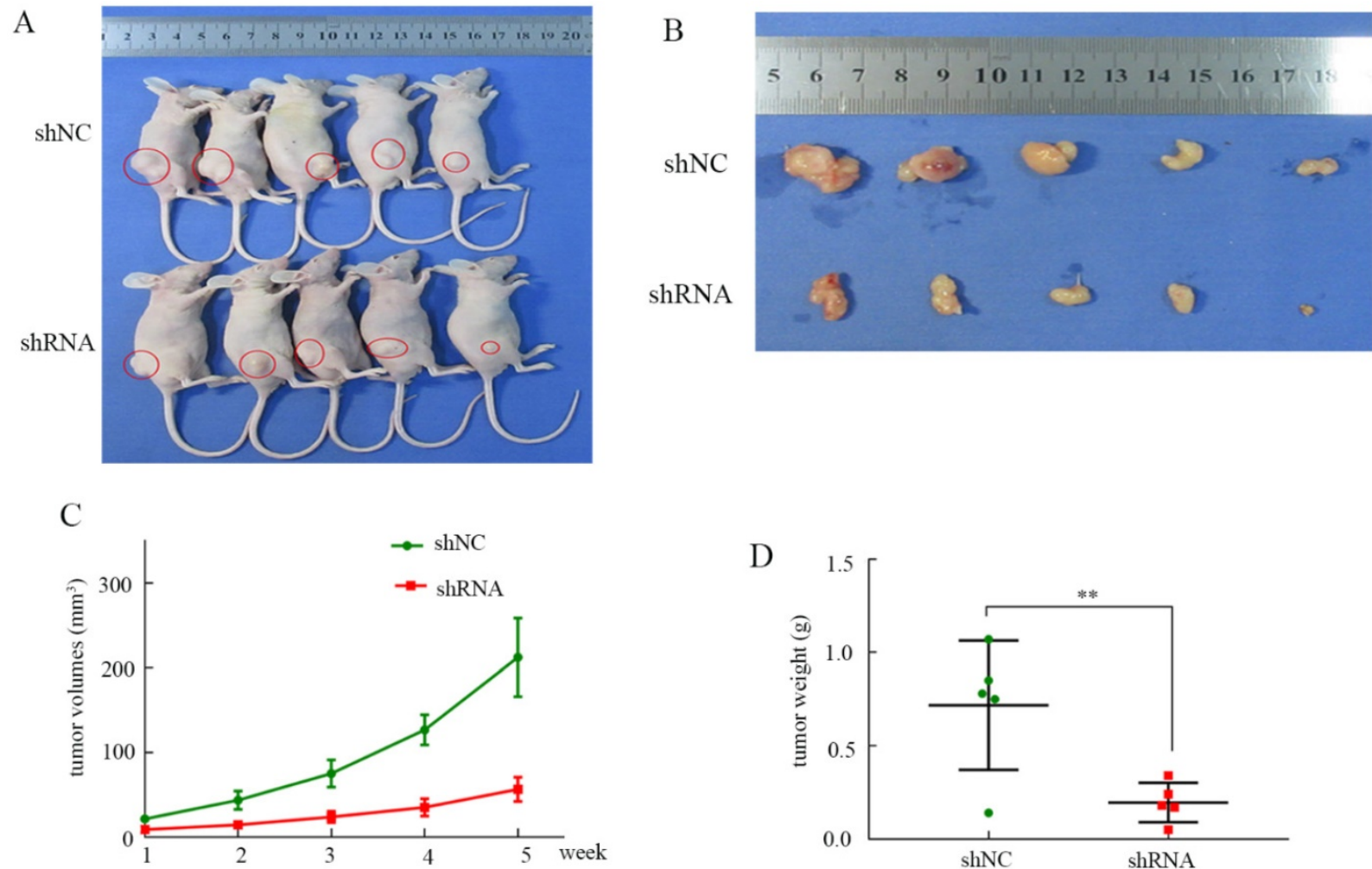

Figure 8. Tumor growth in node mice. (A) Tumor growth in node mice receiving BGC-823. (B) Tumor shape of shRNA and shNC groups after disection. (C) The tumor growth curve in shRNA and shNC groups. (D) Tumor weights in shNC and shRNA groups.

$\mathrm{D}$

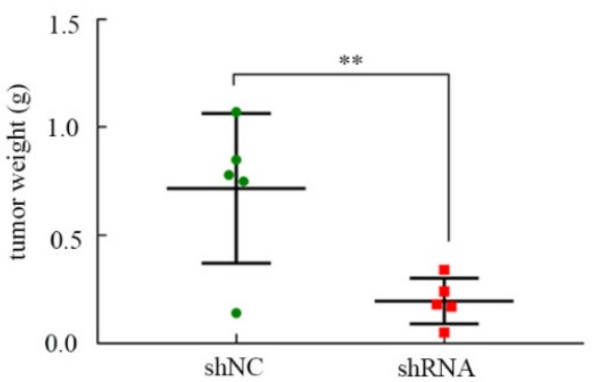


As an oncogene, RNF114 can promote ubiquitylation and degradation of various essential tumor suppressor proteins, while the relative mechanisms of RNF114 are still diminished. Han et al. [44] verified that RNF114 ubiquitinated $\mathrm{p} 21^{\mathrm{WAF} 1}$ and destabilized p27KIP1 and P57KIP2, whose alterations may be implicated in human tumors. Interestingly, RNF114 may play a crucial assistive role in activating EGR1 ubiquitylation and degradation. The role of EGR1 in GC is reportedly controversial. As described by Zhonghua [45], EGR1 critically activated linc01503, which was significantly elevated and remarkably linked to overall survival in GC. Hai-Ting et al. [46] suggested that overexpression of EGR1 enhanced cell proliferation and the cell cycle by mediating transcription of lncRNA-HNF1A-AS1, contributing to GC progression. However, Crawford et al. [47] suggested that EGR1 can interact with TBX2 to co-repress EGR1-target gene expression and is a tumor suppressor in breast cancer. The expression of p21 and its downstream molecules involved in apoptosis were inhibited by EGR1 silencing in GC [21]. Guangda found that the low expression of EGR1 is a hub gene and is related to the prognosis of HER2-positive GC [48]. The results summarized above suggest that the role of EGR1 remains unclear and needs to be studied further in GC.

In a recent study $[49,50]$, nimbolide was shown to recruit and inactivate RNF114 covalent modifications via cysteine-8 (C8) binding in RNF114, thereby protecting tumor suppressors from ubiquitylation. Rodriguez [51] and Liu [52] found that RNF114 is a new partner of A20, which activates nuclear factor-B (NF-B) and modulates the $T$ cellmediated immune response. Boren [53] also proposed that RNF114 regulates various cell biological functions, such as cellular dsRNA responses, cell cycle progression, NF-KB activity, and T-cell activation. Based on the above RNF114 research, we may have discovered new therapies for GC, such as targeting or immunotherapy drugs. These therapies require further in-depth research before they can be used clinically.

Interestingly, in the Lin study [54], RNF114 may be a potential target gene of miR-338-3p, and enhancing miR-338-3p could distinctly inhibit RNF114 expression with pemphigus. In our study, we predicted that hsa-miR-218-5p may be a potential regulator of RNF114 in GC. The study involving hsa-miR-218-5p specifically targeted the 3'-UTR regions of CDK6, cyclin D1, and BIRC5 to suppress GC development [24,55]. In contrast, Xiaolin et al. [56] found that SP1 acts as a transcription factor that binds to the DNA transcriptional chain of RNF114 to control transcription levels. A correlation between RNF114 and SP1 was found in our study. Combining Xiaolin's study, we speculate that SP1 controls RNF114 transcription levels by acting as a transcription factor. RNF114 gene methylation has not been reported; this study is the first to explore the association between RNF114 methylation and GC patient prognosis. The divergence with RNF114 methylation was associated with probe corresponding methylated CpG sites. Not only the methylation levels but also the overall prognosis of GC patients differs between the different probes. The methylation status of the RNF114 promoter was determined by MSP, however, this function of methylation needs further investigation.

Overall, this research suggests that RNF114 may be a pivotal factor in GC. Regrettably, our team did not further elucidate the upstream regulatory mechanism and downstream ubiquitin-binding proteins via experiments. Therefore, further research is needed to elucidate the role of RNF114 and its molecular mechanism in GC.

\section{Acknowledgements}

This work was supported by the National Natural Science Foundation of China (No. 81860428), the Youth Science Foundation of Jiangxi Province (No. 20202BABL216051), the Science and Technology Plan of Health Commission of Jiangxi Province (No. 20191026), the Spark Promotion Plan of Grassroots Health Appropriate Technology of the Health Commission of Jiangxi Province (No. 20198012), the Project of Science and Technology Department of Jiangxi Province (No. 20203BBGL73187), and Traditional Chinese Medicine Research Project of Jiangxi Province (No. 2019A185). Youth Talent Training Program of the First Affiliated Hospital of Nanchang University (No. YFYPY202007).

\section{Ethics Committee Approval and Patient Consent}

Written informed consent was obtained from all patients. All experiments involved in this study were approved by the Ethics Committee of the First Affiliated Hospital of Nanchang University, and the ethical approval number was 015 .

\section{Author Contributions}

Zongfeng Feng, Leyan Li, Qingwen Zeng and Yang Zhang participated in this study and drafted the manuscript. Yi Tu, Wenzheng Chen, Xufeng Shu, and Ahao Wu conducted data analysis and participated in study. Zhengrong $\mathrm{Li}$ and $\mathrm{Yi}$ Cao and Jianbo Xiong designed this study and revised the manuscript. 


\section{Competing Interests}

The authors have declared that no competing interest exists.

\section{References}

[1] Sung H, Ferlay J, Siegel RL et al. Global cancer statistics 2020: GLOBOCAN estimates of incidence and mortality worldwide for 36 cancers in 185 countries. CA Cancer J Clin. 2021.

[2] Van Cutsem E, Sagaert X, Topal B et al. Gastric cancer. Lancet. 2016; 388: 2654-2664.

[3] Yu J, Huang C, Sun Y et al. Effect of Laparoscopic vs Open Distal Gastrectomy on 3-Year Disease-Free Survival in Patients with Locally Advanced Gastric Cancer: The CLASS-01 Randomized Clinical Trial. Jama. 2019; 321: 1983-1992.

[4] Ma YX, Zhang SZ, Hou YP et al. Identification of a novel human zinc finger protein gene ZNF313. Sheng $\mathrm{Wu}$ Hua Xue Yu Sheng $\mathrm{Wu} \mathrm{Wu} \mathrm{Li}$ Xue Bao (Shanghai). 2003; 35: 230-237.

[5] Capon F, Bijlmakers MJ, Wolf N et al. Identification of ZNF313/RNF114 as a novel psoriasis susceptibility gene. Hum Mol Genet. 2008; 17: 1938-1945.

[6] Wang D, Zhu ZZ, Jiang H et al. Multiple genes identified as targets for 20q13.12-13.33 gain contributing to unfavorable clinical outcomes in patients with hepatocellular carcinoma. Hepatol Int. 2015; 9: 438-446.

[7] Bijlmakers MJ, Kanneganti SK, Barker JN et al. Functional analysis of the RNF114 psoriasis susceptibility gene implicates innate immune responses to double-stranded RNA in disease pathogenesis. Hum Mol Genet. 2011; 20: 3129-3137.

[8] $\mathrm{Li} \mathrm{N}$, Sun $\mathrm{H}, \mathrm{Wu} \mathrm{Q}$ et al. Cloning and expression analysis of a novel mouse zinc finger protein gene Znf313 abundantly expressed in testis. J Biochem Mol Biol. 2007; 40: 270-276.

[9] Sheng $Y$, Jin $X, X u$ J et al. Sequencing-based approach identified three new susceptibility loci for psoriasis. Nat Commun. 2014; 5: 4331.

[10] Villarreal-Martinez A, Gallardo-Blanco H, Cerda-Flores $\mathrm{R}$ et al. Candidate gene polymorphisms and risk of psoriasis: A pilot study. Exp Ther Med. 2016; 11: 1217-1222.

[11] Chandran V. The genetics of psoriasis and psoriatic arthritis. Clin Rev Allergy Immunol. 2013; 44: 149-156.

[12] Lin B, Ke Q, Leaman DW et al. Regulation of RANKL-induced osteoclastogenesis by RING finger protein RNF114. J Orthop Res. 2018; 36: 159-166.

[13] Huang S, Li Y, Yuan X et al. The UbL-UBA Ubiquilin4 protein functions as a tumor suppressor in gastric cancer by p53-dependent and p53-independent regulation of p21. Cell Death Differ. 2019; 26: 516-530.

[14] Narayan G, Murty VV. Integrative genomic approaches in cervical cancer: implications for molecular pathogenesis. Future Oncol. 2010; 6: 1643-1652.

[15] Yang M, Teng W, Qu Y et al. Sulforaphene inhibits triple negative breast cancer through activating tumor suppressor Egr1. Breast Cancer Res Treat. 2016; 158: 277-286.

[16] Mohamad T, Kazim N, Adhikari A et al. EGR1 interacts with TBX2 and functions as a tumor suppressor in rhabdomyosarcoma. Oncotarget. 2018; 9: 18084-18098.

[17] Yan L, Wang Y, Liang J et al. MiR-301b promotes the proliferation, mobility, and epithelial-to-mesenchymal transition of bladder cancer cells by targeting EGR1. Biochem Cell Biol. 2017; 95: 571-577.

[18] $\mathrm{Li} \mathrm{L}$, Ameri $\mathrm{AH}$, Wang $\mathrm{S}$ et al. EGR1 regulates angiogenic and osteoclastogenic factors in prostate cancer and promotes metastasis. Oncogene. 2019; 38: 6241-6255.

[19] Feng YH, Su YC, Lin SF et al. Oct4 upregulates osteopontin via Egr1 and is associated with poor outcome in human lung cancer. BMC Cancer. 2019; 19: 791.

[20] Yang Y, Wu F, Zhang J, et al. "EGR1 interacts with DNMT3L to inhibit the transcription of miR-195 and plays an anti-apoptotic role in the development of gastric cancer. J Cell Mol Med. 2019; 23: 7372-7381.

[21] Ko H, Kim JM, Kim SJ et al. Induction of apoptosis by genipin inhibits cell proliferation in AGS human gastric cancer cells via Egr1/p21 signaling pathway. Bioorg Med Chem Lett. 2015; 25: 4191-4196.

[22] Wang H, Zhan M, Xu SW et al. miR-218-5p restores sensitivity to gemcitabine through PRKCE/MDR1 axis in gallbladder cancer. Cell Death Dis. 2017; 8: e2770.

[23] Zhang T, Beeharry MK, Wang Z et al. YY1-modulated long non-coding RNA SNHG12 promotes gastric cancer metastasis by activating the miR-218-5p/YWHAZ axis. Int J Biol Sci. 2021; 177: 1629-1643.

[24] Deng M, Zeng C, Lu X et al. miR-218 suppresses gastric cancer cell cycle progression through the CDK6/Cyclin D1/E2F1 axis in a feedback loop. Cancer Lett. 2017; 403: 175-185.
[25] Li T, Fan J, Wang B et al. TIMER: A Web Server for Comprehensive Analysis of Tumor-Infiltrating Immune Cells. Cancer Res. 2017; 77: e108-e110.

[26] Tang Z, Li C, Kang B et al. GEPIA: a web server for cancer and normal gene expression profiling and interactive analyses. Nucleic Acids Res. 2017; 45: W98-w102.

[27] Szász AM, Lánczky A, Nagy Á et al. Cross-validation of survival associated biomarkers in gastric cancer using transcriptomic data of 1,065 patients. Oncotarget. 2016; 7: 49322-49333.

[28] Modhukur V, Iljasenko T, Metsalu T et al. MethSurv: a web tool to perform multivariable survival analysis using DNA methylation data. Epigenomics. 2018; 10: 277-288.

[29] Zhou G, Soufan O, Ewald J et al. NetworkAnalyst 3.0: a visual analytics platform for comprehensive gene expression profiling and meta-analysis. Nucleic Acids Res. 2019; 47: W234-w241.

[30] $\mathrm{Li} \mathrm{JH}$, Liu S, Zhou $\mathrm{H}$ et al. starBase v2.0: decoding miRNA-ceRNA, miRNA-ncRNA and protein-RNA interaction networks from large-scale CLIP-Seq data. Nucleic Acids Res. 2014; 42: D92-97.

[31] Jiao M, Qi M, Zhang F et al. CUL4B regulates cancer stem-like traits of prostate cancer cells by targeting BMI1 via miR200b/c. Prostate. 2019; 79: 1294-1303.

[32] Yao Y, Liu Z, Guo H et al. Elevated TRIM23 expression predicts poor prognosis in Chinese gastric cancer. Pathol Res Pract. 2018; 214: 2062-2068.

[33] Du Y, Li D, Li N et al. POFUT1 promotes colorectal cancer development through the activation of Notch1 signaling. Cell Death Dis. 2018; 9: 995.

[34] Liu S, Yang Y, Wang W et al. Long noncoding RNA TUG1 promotes cell proliferation and migration of renal cell carcinoma via regulation of YAP. J Cell Biochem. 2018; 119: 9694-9706.

[35] Sun J, Zhang T, Cheng M et al. TRIM29 facilitates the epithelial-tomesenchymal transition and the progression of colorectal cancer via the activation of the Wnt/ beta-catenin signaling pathway. J Exp Clin Cancer Res. 2019; 38: 104

[36] Xia CQ, Han K, Qi Y et al. A Self-Training Subspace Clustering Algorithm under Low-Rank Representation for Cancer Classification on Gene Expression Data. IEEE/ACM Trans Comput Biol Bioinform. 2018; 15: 1315-1324.

[37] Spradlin JN, Hu X, Ward CC et al. Harnessing the anti-cancer natural product nimbolide for targeted protein degradation. Nat Chem Biol. 2019 .

[38] Takahashi-Kanemitsu A, Knight CT, Hatakeyama M. Molecular anatomy and pathogenic actions of Helicobacter pylori CagA that underpin gastric carcinogenesis. Cell Mol Immunol. 2020; 171: 50-63.

[39] Praud D, Rota M, Pelucchi C et al. Cigarette smoking and gastric cancer in the Stomach Cancer Pooling (StoP) Project. Eur J Cancer Prev. 2018; 27: 124-133.

[40] Trujillo Rivera A, Sampieri CL, Morales Romero J et al. Risk factors associated with gastric cancer in Mexico: education, breakfast and chili. Rev Esp Enferm Dig. 2018; 110: 372-379.

[41] Meric-Bernstam F, Johnson AM, Dumbrava EEI et al. Advances in HER2-Targeted Therapy: Novel Agents and Opportunities Beyond Breast and Gastric Cancer. Clin Cancer Res. 2019; 25: 2033-2041.

[42] Sammarco G, Varricchi G, Ferraro V et al. Mast Cells, Angiogenesis and Lymphangiogenesis in Human Gastric Cancer. Int J Mol Sci. 2019; 20, 9.

[43] Li Y, Zhang M, Dorfman RG et al. SIRT2 Promotes the Migration and Invasion of Gastric Cancer through RAS/ERK/JNK/MMP-9 Pathway by Increasing PEPCK1-Related Metabolism. Neoplasia. 2018; 20: 745-756.

[44] Han J, Kim YL, Lee KW et al. ZNF313 is a novel cell cycle activator with an E3 ligase activity inhibiting cellular senescence by destabilizing p21(WAF1.). Cell Death Differ. 2013; 20: 1055-1067.

[45] Ma Z, Gao X, Shuai Y et al. EGR1-mediated linc01503 promotes cell cycle progression and tumorigenesis in gastric cancer. Cell Prolif. 2021; 54: e12922.

[46] Liu HT, Liu S, Liu L et al. EGR1-Mediated Transcription of lncRNA-HNF1A-AS1 Promotes Cell-Cycle Progression in Gastric Cancer. Cancer Res. 2018; 78: 5877-5890.

[47] Crawford NT, McIntyre AJ, McCormick A et al. TBX2 interacts with heterochromatin protein 1 to recruit a novel repression complex to EGR1-targeted promoters to drive the proliferation of breast cancer cells. Oncogene. 2019; 38: 5971-5986.

[48] Yang G, Jian L, Lin X et al. Bioinformatics Analysis of Potential Key Genes in Trastuzumab-Resistant Gastric Cancer. Dis Markers. 2019; 1372571.

[49] Tong B, Spradlin JN, Novaes LFT et al. A Nimbolide-Based Kinase Degrader Preferentially Degrades Oncogenic BCR-ABL. ACS Chem Biol. 2020; 15: 1788-1794

[50] Spradlin JN, Hu X, Ward CC et al. Harnessing the anti-cancer natural product nimbolide for targeted protein degradation. Nat Chem Biol. 2019; 15: 747-755. 
[51] Rodriguez MS, Egana I, Lopitz-Otsoa $\mathrm{F}$ et al. The RING ubiquitin E3 RNF114 interacts with A20 and modulates NF-kappaB activity and T-cell activation. Cell Death Dis. 2014; 5: e1399.

[52] Liu X, Liu Y, Xu M et al. Zinc finger protein A20 is involved in the antipsoriatic effect of calcipotriol. Br J Dermatol. 2016; 175, 2: 314-324.

[53] Lin B, Ke Q, Li H et al. Negative regulation of the RLH signaling by the E3 ubiquitin ligase RNF114. Cytokine. 2017; 99: 186-193.

[54] Lin N, Liu Q, Wang $M$ et al. Usefulness of miRNA-338-3p in the diagnosis of pemphigus and its correlation with disease severity. PeerJ. 2018; 6: e5388.

[55] Zou J, Liao X, Zhang J et al. Dysregulation of miR-195-5p/-218-5p/ BIRC5 axis predicts a poor prognosis in patients with gastric cancer. J Biol Regul Homeost Agents. 2019; 33: 1377-1385.

[56] Liao $X$, Deng W, Lu Y et al. Sp1 plays an important role in regulating the transcription of ZNF313. Cell Biol Int. 2010; 34: 901-905. 